\title{
A Family of Extended NQD Bivariate Distributions with Continuous Marginals
}

\author{
Dae-Hee Ryu ${ }^{1, a}$ \\ ${ }^{a}$ Department of Computer Science, ChungWoon University
}

\begin{abstract}
In this paper we define extended negative quadrant dependence which is weaker negative quadrant dependence and show conditions for having extended negative quadrant dependence property. We also derive generalized Farlie-Gumbel-Morgenstern uniform distributions that possess the extended quadrant dependence property.

Keywords: Positive quadrant dependence, negative quadrant dependence, extended negative quadrant dependence, Farlie-Gumbel-Morgenstern distribution, copula.
\end{abstract}

\section{Introduction}

Lehmann (1966) introduced a natural definition of dependence in the bivariate case. Two random variables $X$ and $Y$ are said to be negatively quadrant dependent(NQD) if for real numbers $x, y, P(X \leq$ $x, Y \leq y) \leq P(X \leq x) P(Y \leq y)$ or $P(X>x, Y>y) \leq P(X>x) P(Y>y)$. Two random variables $X$ and $Y$ are said to be positively quadrant dependent(PQD) if the inequalities above are in the reverse direction. Joag-Dev and Proschan (1983) extended the concept of negative quadrant dependence to the multivariate case. A sequence $\left\{X_{i}, 1 \leq i \leq n\right\}$ of random variables is said to be negatively upper orthant dependent(NUOD) if for all real numbers $x_{1}, \ldots, x_{n}$,

$$
P\left(X_{1}>x_{1}, \ldots, X_{n}>x_{n}\right) \leq \prod_{i=1}^{n} P\left(X_{i}>x_{i}\right)
$$

and it is said to be negatively lower orthant dependent(NLOD) if for all real numbers $x_{1}, \ldots, x_{n}$,

$$
P\left(X_{1} \leq x_{1}, \ldots, X_{n} \leq x_{n}\right) \leq \prod_{i=1}^{n} P\left(X_{i} \leq x_{i}\right) .
$$

A sequence $\left\{X_{i}, 1 \leq i \leq n\right\}$ of random variables is said to be negatively orthant dependent(NOD) if both (1.1) and (1.2) hold.

Recently, Liu (2009) introduced the concept of extended negative dependence in the multivariate case. A sequence $\left\{X_{i}, 1 \leq i \leq n\right\}$ of random variables is said to be extended negatively upper orthant dependent(extended NUOD) if for all real numbers $x_{1}, \ldots, x_{n}$, there exists a constant $M>0$ such that

$$
P\left(X_{1}>x_{1}, \ldots, X_{n}>x_{n}\right) \leq M \prod_{i=1}^{n} P\left(X_{i}>x_{i}\right) .
$$

\footnotetext{
${ }^{1}$ Professor, Department of Computer Science, ChungWoon University, Chungnam 351-701, Korea.

E-mail: rdh@chungwoon.ac.kr
} 
and it is said to be extended negatively lower orthant dependent(extended NLOD)if for all real numbers $x_{1}, \ldots, x_{n}$, there exists a constant $M>0$ such that

$$
P\left(X_{1} \leq x_{1}, \ldots, X_{n} \leq x_{n}\right) \leq M \prod_{i=1}^{n} P\left(X_{i} \leq x_{i}\right) .
$$

A sequence $\left\{X_{i}, 1 \leq i \leq n\right\}$ of random variables is said to be extended negatively orthant dependent(extended NOD) if it is both extended NUOD and extended NLOD.

From definitions of NQD and extended NOD we consider the concept of extended negative dependence in the bivariate case as follows.

Definition 1. Two random variables $X$ and $Y$ are said to be extended negatively quadrant dependent(extended NQD) if there exists a constant $M>0$ such that, for all real numbers $x, y$,

$$
P(X \leq x, Y \leq y) \leq M P(X \leq x) P(Y \leq y)
$$

or

$$
P(X>x, Y>y) \leq M P(X>x) P(Y>y) .
$$

Remark 1. (1.5) and (1.6) are equivalent(see Lemma 1 in Section 2). Consider a special case of the Farlie-Gumbel-Morgenstern bivariate distribution of $X$ and $Y$ where both marginals are exponential.

$$
H(x, y)=\left(1-e^{-\lambda_{1} x}\right)\left(1-e^{-\lambda_{2} y}\right)\left[1+\rho e^{-\lambda_{1} x-\lambda_{2} y}\right], \quad-1 \leq \rho \leq 1, \lambda_{1}, \lambda_{2}>0,
$$

(see Johnson and Kotz (1972, pp. 262-263)). When $-1 \leq \rho \leq 0$ it is clear that $X$ and $Y$ are extended NQD as well as NQD and when $0 \leq \rho \leq 1$ they are extended NQD as well as PQD. In other words, when $-1 \leq \rho \leq 1$ they are extended NQD.

Recall that two random variables $X$ and $Y$ are called NQD if (1.5) or (1.6) holds when $M=1$, they are called positively quadrant dependent(PQD) if the inequality (1.5) or (1.6) holds in the reverse direction when $M=1$. Obviously, the NQD random variables must be the extended NQD random variables. On the other hand, for some PQD random variables, it is possible to find a corresponding positive constant $M$ such that (1.5) or (1.6) holds(see the above F-G-M bivariate distribution, when $0 \leq \rho \leq 1)$. Therefore, the extended NQD structure is substantially more comprehensive than the NQD structure in that it can reflect not only a negative quadrant dependence structure but also a positive one, to some extent.

Lai and Xie (2000) studied a class of uniform distribution having positive quadrant dependence property and showed generalized Farlie-Gumbel-Morgenstern distributions which possess the PQD property and Liu (2009) introduced the concept of extended negative dependence by extending the negatively dependent structure.

Inspired by Lai and Xie (2000) and Liu (2009) investigations we define extended negative quadrant dependence and show conditions for having the above extended negative quadrant dependence property. We also show generalized Farlie-Gumbel-Morgenstern copulas that possess its property.

\section{Conditions for Extended Negative Quadrant Dependence}

First we consider some extended negative quadrant dependence properties. 
Lemma 1. Let random variables $X$ and $Y$ be extended $N Q D$. Then, the following (i) and (ii) are equivalent.

(i) There exists a constant $M>0$ such that $P(X \leq x, Y \leq y) \leq M P(X \leq x) P(Y \leq y)$, for all real numbers $x$ and $y$.

(ii) There exists a constant $M>0$ such that $P(X>x, Y>y) \leq M P(X>x) P(Y>y)$ for all real numbers $x$ and $y$.

\section{Proof:}

(i) $\Rightarrow$ (ii): When $P(X>x, Y>y)=0$ obviously (ii) holds.

Now assume that $P(X>x, Y>y) \neq 0$ and (i) holds. If $0<M \leq 1$, then $X$ and $Y$ are NQD. Hence (ii) follows. If $1<M$, then

$$
\begin{aligned}
P(X>x, Y>y) & =P(X>x)-P(X>x, Y \leq y) \\
& =P(X>x)-P(Y \leq y)+P(X \leq x, Y \leq y) \\
& \leq P(X>x)+P(Y>y)-1+M P(X \leq x) P(Y \leq y) \\
& =P(X>x)+P(Y>y)-1+M(1-P(X>x))(1-P(Y>y)) \\
& \leq M-1+M P(X>x) P(Y>y) \\
& =\left(M+\frac{M-1}{P(X>x) P(Y>y)}\right) P(X>x) P(Y>y) \\
& =M^{\prime} P(X>x) P(Y>y),
\end{aligned}
$$

where $M^{\prime}=M+(M-1) /\{P(X>x) P(Y>y)\} \geq M>1$. Hence (i) implies (ii).

(ii) $\Rightarrow$ (i): When $P(X \leq x, Y \leq y)=0$ obviously (i) holds.

Now assume that $P(X \leq x, Y \leq y) \neq 0$ and (ii) holds. If $0<M \leq 1$, then $X$ and $Y$ are NQD. Hence (i) follows. If $1<M$, then

$$
\begin{aligned}
P(X \leq x, Y \leq y) & =P(X \leq x)-P(X \leq x, Y>y) \\
& =P(X \leq x)-P(Y>y)+P(X>x, Y>y) \\
& \leq P(X \leq x)-P(Y>y)+M P(X>x) P(Y>y) \\
& =P(X \leq x)-1+P(Y \leq y)+M(1-P(X \leq x))(1-P(Y \leq y)) \\
& \leq M-1+M P(X \leq x) P(Y \leq y) \\
& =\left(M+\frac{M-1}{P(X \leq x) P(Y \leq y)}\right) P(X \leq x) P(Y \leq y) \\
& =M^{\prime} P(X \leq x) P(Y \leq y),
\end{aligned}
$$

where $M^{\prime}=M+(M-1) /\{P(X \leq x) P(Y \leq y)\} \geq M>1$. Hence (ii) implies (i).

Lemma 2. (Liu, 2009) Let two random variables $X$ and $Y$ be extended $N Q D$. Then,

(i) if $f$ and $g$ are both nondecreasing(or both nonincreasing) functions, then $f(X)$ and $g(Y)$ are extended $N Q D$, 
(ii) if $X$ and $Y$ are nonnegative random variables, then there exists a constant $M>0$ such that $E(X Y) \leq M E X E Y$

(iii) especially, there exists a constant $M>0$ such that for any real number $h, E\left(e^{h(X+Y)}\right) \leq M E\left(e^{h X}\right)$ $E\left(e^{h Y}\right)$.

Definition 2. Let $H(x, y)$ denote the bivariate distribution of $(X, Y)$ having continuous marginal distribution functions $F(x)$ and $G(y)$. The bivariate distribution $H(x, y)$ is said to be extended NQD if there exists a constant $M>0$ such that

$$
H(x, y) \leq M F(x) G(y) \quad \text { or } \quad \bar{H}(x, y) \leq M \bar{F}(x) \bar{G}(y), \quad \text { for all } x, y,
$$

where $\bar{H}(x, y)=P(X>x, Y>y), \bar{F}(x)=1-F(x)$, and $\bar{G}(y)=1-G(y)$.

Example 1. (Farlie, 1960) Farlie-Gumbel-Morgenstern Bivariate Distribution

$$
H(x, y)=F(x) G(y)[1+\rho(1-F(x))(1-G(y))], \quad-1 \leq \rho \leq 1,
$$

or

$$
\bar{H}(x, y)=\bar{F}(x) \bar{G}(y)[1+\rho(1-\bar{F}(x))(1-\bar{G}(y))] .
$$

Note that if $0 \leq \rho \leq 1$, then $H(x, y)$ is positively quadrant dependent; if $-1 \leq \rho \leq 0$, then $H(x, y)$ is NQD; if $-1 \leq \rho \leq 1$, then $H(x, y)$ is extended NQD.

Theorem 1. Every convex combination of two extended NQD bivariate distribution functions having fixed marginal distributions $F(x)$ and $G(y)$ is still extended NQD.

Proof: Let $H_{1}(x, y)$ and $H_{2}(x, y)$ be extended NQD, i.e.,

$$
\begin{array}{ll}
H_{1}(x, y) \leq M_{1} F(x) G(y), & \text { for some } M_{1}>0 \\
H_{2}(x, y) \leq M_{2} F(x) G(y), & \text { for some } M_{2}>0 .
\end{array}
$$

Then, for $0<\alpha<1$

$$
\begin{aligned}
H(x, y) & =\alpha H_{1}(x, y)+(1-\alpha) H_{2}(x, y) \\
& \leq\left(\alpha M_{1}+(1-\alpha) M_{2}\right) F(x) G(y) \\
& =M F(x) G(y),
\end{aligned}
$$

where $M=\alpha M_{1}+(1-\alpha) M_{2} \geq \min \left(M_{1}, M_{2}\right)>0$. Hence, $H(x, y)$ is still extended NQD, i.e., the proof of theorem is complete.

The following theorem gives the conditions for having the extended NQD property.

Theorem 2. Let $H(x, y)$ denote the distribution function of $(X, Y)$ having continuous marginal distribution functions $F(x)$ and $G(y)$ with marginal probability density functions $f=F^{\prime}, g=G^{\prime}$. Assume that $H(x, y)$ may be written as

$$
H(x, y)=F(x) G(y)+w(x, y), \quad \text { for all } x \text { and } y,
$$


with $w(x, y)$ satisfying the following conditions;

$$
\begin{aligned}
& \text { there exists a constant } m>0 \text { such that } \quad w(x, y) \leq(m-1) F(x) G(y), \\
& w(x, \infty)=0, \quad w(\infty, y)=0, \quad w(x,-\infty)=0, \quad w(-\infty, y)=0, \quad \text { for all } x \text { and } y, \\
& \frac{\partial^{2} w(x, y)}{\partial x \partial y}+f(x) g(y) \geq 0, \quad \text { for all } x \text { and } y .
\end{aligned}
$$

Then $H(x, y)$ is extended $N Q D$.

Proof: The proof is obtained easily.

Remark 2. Consider a special case of the $F-G-M$ system where both marginal distribution functions are exponential. $H(x, y)$ and $\bar{H}(x, y)$ are forms in Example 1, see for example, Johnson and Kotz (1972)

$$
\begin{aligned}
& H(x, y)=\left(1-e^{-\lambda_{1} x}\right)\left(1-e^{-\lambda_{2} y}\right)\left[1+\rho e^{-\lambda_{1} x-\lambda_{2} y}\right], \quad-1 \leq \rho \leq 1, \lambda_{1}, \lambda_{2}>0, \\
& \bar{H}(x, y)=e^{-\lambda_{1} x-\lambda_{2} y}\left[1+\rho\left(1-e^{-\lambda_{1} x}\right)\left(1-e^{-\lambda_{2} y}\right)\right] .
\end{aligned}
$$

Clearly, for $-1 \leq \rho \leq 1 w(x, y)=\rho e^{-\lambda_{1} x-\lambda_{2} y}\left(1-e^{-\lambda_{1} x}\right)\left(1-e^{-\lambda_{2} y}\right)$ satisfies conditions (2.3), (2.4) and (2.5). Moreover, if $0 \leq \rho \leq 1$ then $H(x, y)$ is positively quadrant dependent; if $-1 \leq \rho \leq 0$ then $H(x, y)$ is negatively quadrant dependent; and if $-1 \leq \rho \leq 1$ then $H(x, y)$ is extended negatively quadrant dependent.

Theorem 3. Every convex combination of two bivariate distribution functions which have fixed marginals $F(x), G(y)$ and satisfy conditions (2.3), (2.4) and (2.5) still satisfies conditions (2.3), (2.4) and (2.5).

\section{Proof: Let}

$$
H_{1}(x, y)=F(x) G(y)+w_{1}(x, y), \quad H_{2}(x, y)=F(x) G(y)+w_{2}(x, y),
$$

where $w_{1}(x, y)$ and $w_{2}(x, y)$ satisfy conditions (2.3), (2.4) and (2.5).

Then

$$
H(x, y)=\alpha H_{1}(x, y)+(1-\alpha) H_{2}(x, y) .
$$

Hence, by (2.6) and (2.7) we have

$$
H(x, y)=F(x) G(y)+w(x, y),
$$

where $w(x, y)=\alpha w_{1}(x, y)+(1-\alpha) w_{2}(x, y)$.

Clearly, $w(x, y)$ satisfies condition (2.4). We also obtain

$$
\begin{aligned}
\frac{\partial^{2} w(x, y)}{\partial x \partial y}+f(x) g(y) & =\alpha \frac{\partial^{2} w_{1}(x, y)}{\partial x \partial y}+(1-\alpha) \frac{\partial^{2} w_{2}(x, y)}{\partial x \partial y}+\alpha f(x) g(y)+(1-\alpha) f(x) g(y) \\
& =\alpha\left[\frac{\partial^{2} w_{1}(x, y)}{\partial x \partial y}+f(x) g(y)\right]+(1-\alpha)\left[\frac{\partial^{2} w_{2}(x, y)}{\partial x \partial y}+f(x) g(y)\right] \geq 0 .
\end{aligned}
$$


Hence, $w(x, y)$ satisfies condition (2.5). Finally by Theorem 2 we have

$$
\begin{aligned}
w(x, y) & =\alpha w_{1}(x, y)+(1-\alpha) w_{2}(x, y) \\
& \leq \alpha(m-1) F(x) G(y)+(1-\alpha)(m-1) F(x) G(y)=(m-1) F(x) G(y),
\end{aligned}
$$

which satisfies condition (2.3). Hence, $H(x, y)$ is an extended NQD bivariate distribution satisfying conditions (2.3), (2.4) and (2.5).

\section{A Generalized Farlie-Gumbel-Morgenstern Copula}

For simplicity, let $F$ and $G$ be continuous and let $X$ and $Y$ be dependent according to a copula $C(u, v)$ for $0 \leq u \leq 1,0 \leq v \leq 1$. Thus the joint distribution of $X$ and $Y$ is given by

$$
H(x, y)=C(F(x), G(y))
$$

(see, e.g. Nelsen (2006, p.15)). Let $U=F(X)$ and $V=G(Y)$, so that they are two uniform random variables following the joint distribution $C(u, v)=H\left(F^{-1}(u), G^{-1}(v)\right)$, where $F^{-1}(u)=\inf \{x: F(x)=$ $u$, i.e., $F^{-1}(u)$ is either the inverse function of $F$ or the inverse set function. $G^{-1}(v)$ is defined similarly.

It is well known that the $F-G-M$ bivariate distribution discussed in Section 2, Example 1 has copula given by

$$
C(u, v)=u v\{1+\rho(1-u)(1-v)\}, \quad-1 \leq \rho \leq 1 .
$$

For $-1 \leq \rho \leq 1,(3.2)$ gives rise to an extended NQD bivariate distribution with $w(u, v)=\rho u v(1-$ $u)(1-v)$.

Let $w(u, v)$ be generalized further to a bivariate beta function, i.e.,

$$
w(u, v)=\rho u^{b} v^{b}(1-u)^{a}(1-v)^{a}, \quad a \geq 1, b \geq 1,-1 \leq \rho \leq 1 .
$$

Now we consider a new family of extended NQD bivariate distributions from (3.3). The main result is given in the following theorem and its proof is in the appendix.

Theorem 4. Let $C(u, v)=u v+w(u, v)=u v+\rho u^{b} v^{b}(1-u)^{a}(1-v)^{a}, a \geq 1, b \geq 1,-1 \leq \rho \leq 1$. Then, $C(u, v)$ is the distribution function of a bivariate uniform distribution having the extended NQD property, i.e, $w(u, v)$ satisfies conditions (2.3), (2.4) and (2.5).

Proof: It is clear that conditions (2.3) and (2.4) are satisfied. The condition (2.5) is also satisfied by Appendix B.

Corollary 1. Suppose that $w(u, v)$ has a form

$$
w(u, v)=\rho u v(1-u)^{a}(1-v)^{a}, \quad a \geq 1,-1 \leq \rho \leq 1 .
$$

Then,

$$
C(u, v)=u v\left\{1+\rho(1-u)^{a}(1-v)^{a}\right\}
$$

gives rise to an extended NQD bivariate distribution.

Remark 3. Note that if $0 \leq \rho \leq 1$, then $C(u, v)$ is PQD and if $-1 \leq \rho \leq 0$, then $C(u, v)$ is NQD. 
Theorem 5. Let $C_{1}(u, v)=u v+\rho_{1} u^{b_{1}} v^{b_{1}}(1-u)^{a_{1}}(1-v)^{a_{1}}, a_{1} \geq 1, b_{1} \geq 1,-1 \leq \rho_{1} \leq 1$ and $C_{2}(u, v)=u v+\rho_{1} u^{b_{2}} v^{b_{2}}(1-u)^{a_{2}}(1-v)^{a_{2}}, a_{2} \geq 1, b_{2} \geq 1,-1 \leq \rho_{2} \leq 1$ be the distributions of bivariate uniform distribution, and the convex combination $C(u, v)=\alpha C_{1}(u, v)+(1-\alpha) C_{2}(u, v)$, $0<\alpha<1$. Then, if both $C_{1}(u, v)$ and $C_{2}(u, v)$ satisfy conditions (2.3), (2.4) and (2.5), then $C(u, v)$ is the distribution of a bivariate uniform distribution having extended NQD property, that is, (2.3), (2.4) and (2.5) are satisfied.

\section{Proof:}

(i) The case $a_{1}=a_{2}=a, b_{1}=b_{2}=b$; Let

$$
\begin{aligned}
C(u, v) & =\alpha C_{1}(u, v)+(1-\alpha) C_{2}(u, v) \\
& =u v+\left[\alpha \rho_{1}+(1-\alpha) \rho_{2}\right] u^{b} v^{b}(1-u)^{a}(1-v)^{a} \\
& =u v+\rho u^{b} v^{b}(1-u)^{a}(1-v)^{a} \\
& =u v+w(u, v), \quad \text { where } \rho=\alpha \rho_{1}+(1-\alpha) \rho_{2} .
\end{aligned}
$$

Note that if $-1 \leq \rho_{1} \leq 1$ and $-1 \leq \rho_{2} \leq 1$, then $-1 \leq \rho \leq 1$.

Hence, by Theorem 4, when $a=a_{1}=a_{2}, b=b_{1}=b_{2}$, if $-1 \leq \rho_{1} \leq 1$ and $-1 \leq \rho_{2} \leq 1$, then $w(u, v)$ satisfies conditions (2.3), (2.4) and (2.5), that is, $C(u, v)$ is extended NQD.

(ii) The case $a_{1} \neq a_{2}, b_{1} \neq b_{2}$; Let

$$
\begin{aligned}
C(u, v) & =\alpha C_{1}(u, v)+(1-\alpha) C_{2}(u, v) \\
& =u v+\alpha \rho_{1} u^{b_{1}} v^{b_{1}}(1-u)^{a_{1}}(1-v)^{a_{1}}+(1-\alpha) \rho_{2} u^{b_{2}} v^{b_{2}}(1-u)^{a_{2}}(1-v)^{a_{2}} \\
& =u v+w(u, v) .
\end{aligned}
$$

By Theorem 4 there exist constants $m_{1}>0$ and $m_{2}>0$ such that $\rho_{1} u^{b_{1}} v^{b_{1}}(1-u)^{a_{1}}(1-v)^{a_{1}} \leq\left(m_{1}-1\right) u v$ and $\rho_{2} u^{b_{2}} v^{b_{2}}(1-u)^{a_{2}}(1-v)^{a_{2}} \leq\left(m_{2}-1\right) u v$.

Hence, we obtain

$$
\begin{aligned}
w(u, v) & =\alpha \rho_{1} u^{b_{1}} v^{b_{1}}(1-u)^{a_{1}}(1-v)^{a_{1}}+(1-\alpha) \rho_{2} u^{b_{2}} v^{b_{2}}(1-u)^{a_{2}}(1-v)^{a_{2}} \\
& \leq \alpha\left(m_{1}-1\right) u v+(1-\alpha)\left(m_{2}-1\right) u v \\
& \leq(m-1) u v, \quad \text { where } m=\max \left(m_{1}, m_{2}\right)
\end{aligned}
$$

which satisfies (2.3). It is clear that $w(u, v)$ satisfies (2.4).

By Appendix B, for $-1 \leq \rho_{1} \leq 1$ and $-1 \leq \rho_{2} \leq 1$,

$$
\begin{aligned}
\frac{\partial^{2}}{\partial u \partial v}\left[\alpha \rho_{1} u^{b_{1}} v^{b_{1}}(1-u)^{a_{1}}(1-v)^{a_{1}}\right] & \geq-\alpha, \\
\frac{\partial^{2}}{\partial u \partial v}\left[(1-\alpha) \rho_{2} u^{b_{2}} v^{b_{2}}(1-u)^{a_{2}}(1-v)^{a_{2}}\right] & \geq-(1-\alpha) .
\end{aligned}
$$

Hence, $\partial^{2} w(u, v) / \partial u \partial v \geq-1$ for $-1 \leq \rho_{1} \leq 1$ and $-1 \leq \rho_{2} \leq 1$, which satisfies (2.5).

Hence, $w(u, v)$ satisfies (2.3), (2.4) and (2.5).

\section{Appendix A:}

From Theorem 2 we obtain the conditions that the copula family has the extended NQD property. 
Theorem 6. A bivariate copula $C(u, v)$ may be written as $C(u, v)=u v+w(u, v), 0 \leq u, v \leq 1$, satisfying the following conditions:

(i) $w(u, 1)=w(1, v)=w(u, 0)=w(0, v)=0$, for all $0 \leq u, v \leq 1$,

(ii) $\frac{\partial^{2} w(u, v)}{\partial u \partial v} \geq-1, \quad$ for all $0 \leq u, v \leq 1$.

If $w(u, v) \geq(\leq) 0$ for all $0 \leq u, v \leq 1$ then $C(u, v)$ is $P Q D(N Q D)$.

If there exists a constant $M>0$ such that $w(u, v) \leq(M-1) u v$, then $C(u, v)$ is extended $N Q D$.

\section{Mardia family}

$$
\begin{aligned}
C(u, v) & =\frac{\theta^{2}(1+\theta)}{2} \min (u, v)+\left(1-\theta^{2}\right) u v+\frac{\theta^{2}(1-\theta)}{2} \max (u+v-1,0) \\
& =u v+w(u, v)
\end{aligned}
$$

where $w(u, v)=\theta^{2}(1+\theta) / 2 \min (u, v)-\theta^{2} u v+\theta^{2}(1-\theta) / 2 \max (u+v-1,0),-1 \leq \theta \leq 1$ and $0 \leq u, v \leq 1$.

Clearly, condition (i) is satisfied.

(a) case $1: u \leq v, u+v-1 \geq 0$;

$(\mathrm{a}-1) w(u, v)=\frac{\theta^{2}(1+\theta)}{2} u-\theta^{2} u v+\frac{\theta^{2}(1-\theta)}{2}(u+v-1)$,

(a-2) $\frac{\partial w}{\partial u}=\frac{\theta^{2}(1+\theta)}{2}-\theta^{2} v+\frac{\theta^{2}(1-\theta)}{2}=0$,

(a-3) $\frac{\partial w}{\partial v}=-\theta^{2} u+\frac{\theta^{2}(1-\theta)}{2}=0$.

From (a-2) and (a-3) $u=(1-\theta) / 2$ and $v=1$ follows.

(a-4) $\frac{\partial^{2} w}{\partial u \partial v}=-\theta^{2}<0$.

Hence (ii) is satisfied and $w(u, v) \leq w((1-\theta) / 2,1)=0$.

(b) case $2: u \leq v, u+v-1<0$;

(b-1) $w(u, v)=\frac{\theta^{2}(1+\theta)}{2} u-\theta^{2} u v$,

(b-2) $\frac{\partial w}{\partial u}=\frac{\theta^{2}(1+\theta)}{2}-\theta^{2} v=0$,

(b-3) $\frac{\partial w}{\partial v}=-\theta^{2} u=0$.

From (b-2) and (b-3) $u=0$ and $v=(1+\theta) / 2$.

$$
\frac{\partial^{2} w}{\partial u \partial v}=-\theta^{2}<0
$$


Hence (ii) is satisfied and $w(u, v) \leq w(0,(1+\theta) / 2)=0$.

(c) case $3: u>v, u+v-1 \geq 0$;

By the similar method to (a) (i) and (ii) are satisfied and $w(u, v) \leq w(1,(1-\theta) / 2)=0$.

(d) case $4: u>v, u+v-1 \leq 0$;

By the similar method to (b) (i) and (ii) are satisfied and $w(u, v) \leq w((1+\theta) / 2,0)=0$.

From (a), (b), (c) and (d) $C(u, v)$ is extended NQD.

\section{Ali-Mikhail-Haq family}

$$
C(u, v)=\frac{u v}{1-\theta(1-u)(1-v)}, \quad \theta \in[-1,1),
$$

which is a kind of Archimedean copula. This copula can generate both positive and quadrant dependence structures depending on the sign of $\theta$. If $-1 \leq \theta \leq 0$ then $C(u, v)$ is extended NQD(See Remark 2.4 of Ko and Tang (2008)).

\section{Frank family}

$$
\begin{aligned}
& C(u, v)=-\frac{1}{\theta} \ln \left(1+\frac{\left(e^{-\theta v}-1\right)\left(e^{-\theta u}-1\right)}{e^{-\theta}-1}\right), \quad \theta \geq 0 . \\
& C(u, v)=u v+w(u, v),
\end{aligned}
$$

where $w(u, v)=-u v-1 / \theta \ln \left\{1+\left(e^{-\theta v}-1\right)\left(e^{-\theta u}-1\right) /\left(e^{-\theta}-1\right)\right\}$.

Clearly, (i) is satisfied that is,

$$
w(u, 1)=w(1, v)=w(u, 0)=w(0, v)=0 .
$$

By a tedious calculation we also obtain $\partial^{2} w(u, v) / \partial u \partial v \geq-1$. (ii) is satisfied.

As noted in Remark 3.1 of Ko and Tang (2008) or Example 4.2 of Liu (2009) Frank family belongs to extended NQD category.

\section{Appendix B:}

Lemma 3. Let $w(u, v)=\rho u^{b} v^{b}(1-u)^{a}(1-v)^{a},-1 \leq \rho \leq 1, a, b \geq 1,0 \leq u, v \leq 1$. Then

$$
\frac{\partial^{2} w(u, v)}{\partial u \partial v}=\rho g(u) g(v) \geq-1, \quad \text { for }-1 \leq \rho \leq 1,0 \leq u, v \leq 1,
$$

where

$$
g(u)=u^{b-1}(1-u)^{a-1}[b-(a+b) u] .
$$

Proof: First note that Lai and Xie (2000) had already proved the following result in case $0 \leq \rho \leq 1$. It remains to show that this result still holds in case $-1 \leq \rho \leq 0$, that is, (B.1) holds for $-1 \leq \rho \leq 0$. 
We use the proof appeared in Appendix of Lai and Xie (2000) for $0 \leq \rho \leq 1$. For the completeness we repeat it here. By partial differentiation, we have

$$
\begin{gathered}
\frac{\partial w(u, v)}{\partial u}=\rho v^{b}(1-v)^{a} g(u), \quad \frac{\partial^{2} w(u, v)}{\partial u \partial v}=\rho g(u) g(v) . \\
g^{\prime}(u)=\left\{b(b-1)-2 u[b(a+b-1)]+(a+b)(a+b-1) u^{2}\right\} u^{b-2}(1-u)^{a-2}
\end{gathered}
$$

shows that $g$ is minimum at

$$
u^{*}=\frac{b(a+b-1)+\sqrt{a b(a+b+1)}}{(a+b)(a+b-1)} .
$$

Also, we have

$$
\left[b-(a+b) u^{*}\right]=-\sqrt{\frac{a b}{a+b-1}} .
$$

Now to show that $g(u) \geq-1$, for all $0 \leq u \leq 1$ this requires the following steps:

Step 1: First, we note that $g(u) \geq 0$, for $0 \leq u \leq b /(a+b)$ and $g(u) \leq 0$, for $b /(a+b) \leq u \leq 1$.

In particular, it follows from (B.4) that $b /(a+b) \leq u^{*} \leq 1$ any $g\left(u^{*}\right) \leq 0$.

Step 2: Next, we simplify the algebra in expression for the optimal point $u^{*}$ given in (B.3):

$$
u^{*}=\frac{b(a+b-1)+\sqrt{a b(a+b+1)}}{(a+b)(a+b-1)}=\frac{b}{a+b}+\frac{1}{a+b} \times \sqrt{\frac{a b}{a+b-1}} .
$$

For $a \geq 1, b \geq 1,(b-1) \geq(b-1) / a \Rightarrow a b>a+b-1$ from which $u^{*} \geq(b+1) /(a+b)$.

Step 3: Let $a$ be fixed. Since $(1-u)^{a-1} \geq 0$, we only need to consider $h_{1}(b)=u^{b-1}[b-(a+b) u]$.

Now

$$
h_{1}^{\prime}(b)=u^{b-1}\{\log u \times[b-(a+b) u]+(1-u)\}>0
$$

for $u \geq b /(a+b)$, shows that $g$ is an increasing function in $b$ for $u \geq b /(a+b)$.

Step 4: Let $b$ be fixed. Define $h_{2}(a)=(1-u)^{a-1}[b-(a+b) u]$. We then have $h_{2}^{\prime}(a)=(1-u)^{a-1}\{\log (1-$ $u) \times[b-(a+b) u]-u\} \geq 0$, if $\{\log (1-u)[b-(a+b) u]-u\} \geq 0$.

As $\log (1-u)<-u$, it follows that $\log (1-u) \times[b-(a+b) u]-u>-u[b-(a+b) u]-u>0$, if $u \geq(b+1) /(a+b)$.

In other words, $h_{2}^{\prime}(a) \geq 0$ and there by $g$ is increasing in a for $u \geq(b+1) /(a+b)$. and $b$.

From the preceding four steps, we conclude that at the point $u=u^{*}, g\left(u^{*}\right)$ is increasing in both $a$

Finally, when $a=1$ and $b=1$, we have $g\left(u^{*}\right)=-1$, which implies $g(u) \geq-1$ for all $a \geq 1, b \geq 1$. Also $g(u)<1$ for all $a \geq 1, b \geq 1$ and $0 \leq u \leq 1$.

From the facts that $-1 \leq g(u)<1,-1 \leq g(v)<1$ and $-1 \leq \rho \leq 1$ (B.1) follows. 


\section{Acknowledgments}

The author would like to thank to the referees for their constructive and insightful comments and suggestions that greatly improved the paper.

\section{References}

Farlie, D. J. G. (1960). The performance of some correlation coefficient for a general bivariate distribution, Biometrika, 47, 307-323.

Joag-Dev, K. and Proschan, F. (1983). Negative association of random variables with applications, Annals of Statistics, 11, 286-295.

Johnson, N. L. and Kotz, S. (1972). Distributions in Statistics: Continuous Multivariate Distributions, Wiley, New York.

Ko, B. and Tang, Q. (2008). Sums of dependent nonnegative random variables with subexponential tails, Journal of Applied Probability, 45, 85-94.

Lai, C. D. and Xie, M. (2000). A new family of positive quadrant dependent bivariate distributions, Statistics \& Probability Letters, 46, 359-364.

Lehmann, E. L. (1966). Some concepts of dependence, The Annals of Mathematical Statistics, 37, 1137-1153.

Liu, L. (2009). Precise large deviations for dependent random variables with heavy tails, Statistics \& Probability Letters, 99, 1290-1298.

Nelsen, R. B. (2006). An introduction to Copulas, 2nd, Springer, New York.

Received May 2011; Revised June 2011; Accepted September 2011 